\title{
Retrospective metagenomics from the Dutch NIVEL surveillance network (2006-2017) of 155 sample shows a wide variety of viruses (Coronavirus/Influenza/Enterovirus/Rhinovirus/Adenovirus) and some bacterial pathogens leading to acute respiratory and influenza-like disease
}

\author{
Sandeep Chakraborty
}

\section{Letter}

'Retrospective targeted metagenomics study on nasopharyngeal swab samples from the Dutch NIVEL surveillance network' from 2006 to 2017 of 155 patients has been submitted in the NCBI database (Accid:PRJNA562998). The samples have been classified as either Acute Respiratory Infection (ACI) or Influenza-like Illness (ILI). This dataset is yet to be analyzed in a published paper.

Table 1 shows the number of samples in which each virus family occurs. A chronological view of viruses in circulation in these years are in SI:yearly.txt. The most common is respiratory syncytial virus - causes infections of the lungs and respiratory tract, very common in children, mostly mild - but can cause severe infection in some immunocompromised people [1]. Next, influenza has three types $(\mathrm{A}=17, \mathrm{~B}=13, \mathrm{C}=3)$ (there are bigger issues with influenza with respect to lab-made viruses circulating $[2,3]$ ). This is followed by the endemic human coronaviruses, OC43 being the most common (OC43=14 NL63=7 229E=7 HKU1=4) as seen in 'Human coronavirus circulation in the United States 2014-2017' [4]. Rhinovirus and parainfluenza also commonly cause respiratory illnesses in infants and young children. There is also 2 samples with Rubulavirus which causes mumps, but both are in 2007 - and there are no recent instances of this virus. The Sewage-associated-gemycircularvirus [5] is found in the cerebrospinal fluid from patients with encephalitis [6].

\section{Adenoviruses - not just a common cold virus}

Apart from being associated with the common cold, Adenoviruses are also implicated in transverse myelitis $[7$, 8], which gains importance due the use of adenovirus-vectored vaccines (Oxford) in Covid19. Although replication-deficient, these adenoviruses could easily recombine with the homologous human adenoviruses to generate a novel Ad+Spike virus [9].

\section{Have we really eradicated polio?}

Polio viruses cause fever, headache, sore throat, fatigue, vomiting, back/neck pain or stiffness, muscle weakness or tenderness [10]. While, the exact polio-virus genome may have been (close to been) eradicated, there are several other viruses close to the polio virus - enteroviruses, coxsackieviruses and echoviruses - which cause polio-like paralysis of children. 'Is Enterovirus D68 the new polio? [11] asks a new paper after an 'outbreak of recombinant coxsackievirus A2 infection and polio-like paralysis of children, Taiwan, 2014' [12]. The Polio virus itself was found in sewage samples from the United Kingdom and Senegal, but interestingly not in Pakistan samplescitemajumdar2018environmental, although all enterovirus, coxsackievirus and echovirus was found in all three countries. Also, 'CSFs from adults with meningoencephalitis from a tertiary referral hospital for infectious disease in Ho Chi Minh City, Vietnam during 2016-2017' showed the presence of echoviruses (Accid:PRJNA588650, there is no published study on this yet).

\section{Bacterial read - and bacteriophages}

Several bacterial species are also identified (Bacillus, Escherichia, Haemophilus, Veillonella, Klebsiella, Salmonella, Enterococcus, Streptococcus, Staphylococcus) - and their related phages. The role of bacteria, particularly in those cases where there are no viruses found, need to ascertained. 
Table 1: Viruses in Retrospective metagenomics from the Dutch NIVEL surveillance network (2006-2017) of 155 sample This does not consider co-infections. A wide range of viruses cause similar acute respiratory disease and influenza-like illness. Have we really eradicated polio - since enteroviruses and coxsackieviruses cause polio like symptoms. Also, the effect of adenoviruses needs to be carefully monitored, since several adenovirus-vectored vaccines are in trial for Covid19, and adenoviruses are implicated in transverse myelitis [7,8].

\begin{tabular}{|c|c|c|c|}
\hline Group & Virus & $\mathrm{N}$ & Description \\
\hline 1 & $\begin{array}{l}\text { syncytial } \\
\text { metapneumovirus } \\
\text { orthopneumovirus }\end{array}$ & 33 & $\begin{array}{l}\text { Common respiratory viruses that usually causes mild, cold-like symptoms. } \\
\text { Most people recover in a week or two, but can be serious, especially for infants and older adults. }\end{array}$ \\
\hline 2 & Influenza & 32 & $(\mathrm{~A}=17 \mathrm{~B}=13 \mathrm{C}=3)$ \\
\hline 3 & coronavirus & 28 & $(\mathrm{OC} 43=14 \mathrm{NL} 63=7229 \mathrm{E}=7 \mathrm{HKU} 1=4)$ \\
\hline 4 & $\begin{array}{l}\text { enterovirus } \\
\text { coxsackievirus }\end{array}$ & 15 & $\begin{array}{l}\text { Is Enterovirus D68 the new polio? [11], 'Recombinant Coxsackievirus A2 and Deaths of Children, Hong Kong, 2012' [13] } \\
\text { 'Outbreak of recombinant coxsackievirus A2 infection and polio-like paralysis of children, Taiwan, 2014' [12] }\end{array}$ \\
\hline 5 & rhinovirus & 10 & CDC: Many different respiratory viruses can cause the common cold, but rhinoviruses are the most common \\
\hline 6 & parainfluenza & 7 & CDC: commonly cause respiratory illnesses in infants and young children. \\
\hline 7 & adenovirus & 5 & $\begin{array}{l}\text { Aside from cold, adenoviruses are implicated in transverse myelitis }[7,8] \\
\text { which gains importance due the use of adenovirus-vectored vaccines (Oxford) in Covid19 }\end{array}$ \\
\hline 8 & gemycircularvirus & 4 & $\begin{array}{l}\text { Sewage-associated found in cerebrospinal fluid from patients with encephalitis } \\
\text { Only one genome known from a } 2015 \text { study [5] }\end{array}$ \\
\hline Low & $\begin{array}{l}\text { rubulavirus } \\
\text { herpesvirus } \\
\text { astrovirus } \\
\text { cyclovirus }\end{array}$ & $\begin{array}{l}2 \\
2 \\
1 \\
1\end{array}$ & mumps, 2 samples in 2007 , does not seem to be a recent problem \\
\hline
\end{tabular}




\section{References}

1. Glezen WP, Taber LH, Frank AL, Kasel JA (1986) Risk of primary infection and reinfection with respiratory syncytial virus. American journal of diseases of children 140: 543-546.

2. Chakraborty S (2020). Madin Darby canine kidney cell-lines used in influenza virus production has resulted in canine 28s being integrated in the influenza virus genome - evidence from influenza a patients in hong kong. doi:10.31219/osf.io/wsry4. URL osf .io/wsry4.

3. Chakraborty S (2020). RNA-seq reads of Influenza A genome appended with DNA-expression vector sequences facilitating protein transcription (18s) in Covid19 patients from Wuhan and Hong Kong very worrying as this is unlikely to be contamination. doi:10.31219/osf.io/2dzqy. URL osf .io/2dzqy.

4. Killerby ME, Biggs HM, Haynes A, Dahl RM, Mustaquim D, et al. (2018) Human coronavirus circulation in the united states 2014-2017. Journal of Clinical Virology 101: 52-56.

5. Kraberger S, Argüello-Astorga GR, Greenfield LG, Galilee C, Law D, et al. (2015) Characterisation of a diverse range of circular replication-associated protein encoding dna viruses recovered from a sewage treatment oxidation pond. Infection, Genetics and Evolution 31: 73-86.

6. Zhou Z, Zeng Y, Wu X, Li F, Liu X, et al. (2016) Identification of one bocr mutation and five nf1 mutations in male patients with neurofibromatosis type 1 and congenital pseudarthrosis of the tibia .

7. Memon SB, Shaib F (2008) Adenovirus as a cause of transverse myelitis in the intensive care unit. Chest 134: $36 \mathrm{C}$.

8. Ooi MH, Wong SC, Clear D, Perera D, Krishnan S, et al. (2003) Adenovirus type 21 — associated acute flaccid paralysis during an outbreak of hand-foot-and-mouth disease in sarawak, malaysia. Clinical infectious diseases 36: 550-559.

9. Chakraborty S (2020). Can ChAdOx1, a replication-deficient adenoviral (ad) vector used to deliver SARS-Cov2 spike protein as a vaccine, recombine with the homologous human ad to generate a novel ad+spike virus? doi:10.31219/osf.io/2hj9c. URL osf .io/2hj9c.

10. Chia J, Chia A, Voeller M, Lee T, Chang R (2010) Acute enterovirus infection followed by myalgic encephalomyelitis/chronic fatigue syndrome (me/cfs) and viral persistence. Journal of clinical pathology 63: 165-168.

11. Cassidy H, Poelman R, Knoester M, Leer-Buter V, Coretta C, et al. (2018) Enterovirus d68-the new polio? Frontiers in microbiology 9: 2677.

12. Chiang KL, Wei SH, Fan HC, Chou YK, Yang JY (2019) Outbreak of recombinant coxsackievirus a2 infection and polio-like paralysis of children, taiwan, 2014. Pediatrics \& Neonatology 60: 95-99.

13. Yip CC, Lau SK, Woo PC, Wong SS, Tsang TH, et al. (2013) Recombinant coxsackievirus a2 and deaths of children, hong kong, 2012. Emerging Infectious Diseases 19: 1285. 